Mots. Les langages du politique

\title{
France d'en haut / France d'en bas : Raffarin tout terrain
}

Sylvianne Rémi-Giraud

\section{(2) OpenEdition \\ Journals}

Édition électronique

URL : https://journals.openedition.org/mots/180

DOI : $10.4000 /$ mots. 180

ISSN : 1960-6001

Éditeur

ENS Éditions

Édition imprimée

Date de publication : 1 mars 2005

Pagination : 93-105

ISBN : 2-84788-077-1

ISSN : 0243-6450

Référence électronique

Sylvianne Rémi-Giraud, "France d'en haut / France d'en bas : Raffarin tout terrain », Mots. Les langages du politique [En ligne], 77 | 2005, mis en ligne le 31 janvier 2008, consulté le 22 avril 2022. URL : http:// journals.openedition.org/mots/180; DOI : https://doi.org/10.4000/mots.180 


\section{France d'en haut / France d'en bas : Raffarin tout terrain}

Je propose ici une étude sémantique de l'expression France d'en haut / France d'en bas, orientée dans deux directions : montrer, d'une part, en quoi cette expression a tout particulièrement vocation à s'inscrire dans un discours de proximité et, d'autre part, tenter de dégager une réflexion d'ordre méthodologique sur l'exploitation dans le discours politique de mots hautement polysémiques du lexique courant.

Dès son installation à Matignon, Jean-Pierre Raffarin, dont on sait qu'il a été expert en communication ${ }^{1}$, se fait connaitre comme un ministre amateur de mots et de formules - ces fameuses «raffarinades» dont une certaine presse écrite fut en son temps friande ${ }^{2}$. Si nombre de ces trouvailles, plus ou moins médiatisées, ont fait long feu, l'expression France d'en haut / France d'en bas, qui a connu d'emblée un succès inespéré, poursuit toujours sa course, avec de nombreuses échappées qui la conduisent bien au-delà de ses attaches d'origine, y compris du côté des opposants et détracteurs de la politique gouvernementale. Succès dont se serait sans doute bien passé celui qui en assuma la paternité première ${ }^{3} .$.

1. J.-P. Raffarin a été directeur du marketing de Jacques Vabre, puis, de 1981 à 1988, directeur du département de la communication du cabinet Bernard Krief.

2. Dans Le Monde du 11 mai 2002, A. Defaye et R. Guyotat, dans un article ayant pour titre «En Poitou-Charente, Jean-Pierre Raffarin est apparu comme un homme d'idées et de formules», relèvent, pêle-mêle avec la France d'en bas, les mots gouvernance, [une région] apprenante, périphéricité, maritimité, des maximes ou slogans tels que Soyons ambitieux, mais pas prétentieux, Les bons amis font les bonnes vacances.

3. Paternité non clairement établie, d'ailleurs. On lit dans Les dossiers du Canard enchaîné, $\mathrm{n}^{\circ} 85$, octobre 2002, p. 68, à propos de Dominique Amiel, conseiller chargé de la communication de Raffarin : "Il ne faut guère le pousser, par exemple, pour qu'il revendique comme des tas d'autres être le concepteur de "la France d'en bas” ». Libération (17 mai 2002) va plus loin. Dans un article intitulé " “France d'en bas” : la "récup” de Raffarin. Le Premier ministre se rengorge de sa "trouvaille"... galvaudée depuis Balzac », D. Hassoux fait remonter cette opposition aux Illusions perdues de Balzac décrivant la ville d’Angoulême ("En haut, la noblesse et le pouvoir, en bas, le commerce et l'argent») et en suit la trace chez les écrivains de la droite nationaliste du début du $20^{\mathrm{e}}$ siècle (Maurras, Barrès, Giraudoux, Péguy). Mais surtout, il relève cette expression dans Le Figaro du 18 janvier 1990, sous la plume du politologue J.-L. Parodi («D'un côté, ceux d'en haut, ceux qui nous gouvernent. De l'autre côté, ceux d'en bas, les petits»), soulignant que si J.-P. Raffarin ne possède peut-être pas toutes ces références littéraires, il ne peut ignorer le quotidien de la rue du Louvre...

Université Lyon 2, ICAR-CNRS, remi@univ-lyon2.fr 
Mais rendons à César ce qui appartient à César. On peut dire que l'expression France d'en haut / France d'en bas, telle qu'elle a été employée par Raffarin dans les premiers jours de son ministère, représente un petit tour de force lexical. Si l'on admet qu'au lendemain de l'élection présidentielle de 2002 et de l'état de choc provoqué par la percée de Jean-Marie Le Pen, la France d'en haut désigne prioritairement les dirigeants socialistes au pouvoir et la France d'en bas les couches populaires perdues par la gauche et que la droite gouvernementale entend rallier à sa politique, le premier paradoxe - apparent - est de mettre en position haute (en haut) ses ennemis politiques et en position basse (en bas) ceux à qui l'on s'adresse. Mais ce paradoxe superpose, de manière particulièrement habile, deux points de vue et deux discours. S'il y a une France d'en bas, c'est qu'elle est vue d'en haut - et de haut - par ceux qui ont gouverné la France jusque-là : il s'agit d'un discours d'éloignement, implicitement imputable aux socialistes et que dénonce celui-là même qui s'en fait l'écho. Mais il y a plus. Ce disant, Raffarin affirme dans le même temps sa solidarité avec cette France d'en bas méprisée : c'est là un discours de proximité qu'il tient en son nom propre et qui n'est pas le moindre des paradoxes de la part d'un homme qui occupe la seconde place au sommet de l'État.

On essaiera de montrer comment deux antonymes à première vue très courants et de portée très générale, relevant au départ de l'expérience spatiale et n'ayant pas vocation plus que d'autres à entrer dans le domaine politique, peuvent dire tant de choses en une formule lapidaire et faire mouche dans le débat politique. Pour cela, je proposerai, à partir du matériau que fournit un dictionnaire de langue usuel comme le Nouveau Petit Robert (édition 1993), une étude formelle et sémantique de ces deux unités, qui sont à la fois polycatégorielles, c'est-à-dire susceptibles d'appartenir à des catégories grammaticales différentes (adjectif, nom, adverbe), et fortement polysémiques. J'étudierai cette polysémie en m'attachant tout particulièrement au processus qui conduit des significations spatiales à la multiplicité des significations métaphoriques qui en dérivent. J'accorderai également une grande importance aux représentations (axiologie positive ou négative, valeurs affectives) qui s'attachent aux significations. Pour mener à bien cette étude, j'exploiterai non seulement les définitions proposées par le Petit Robert mais aussi le corpus d'exemples et de citations qui les illustrent. Ce corpus, qui peut aller des expressions (ce bas monde) aux collocations (hauts fonctionnaires, hauts faits) et aux syntagmes libres (une tour assez haute), permet de mettre en place une première grille d'emplois de ce mot, liée à sa combinatoire immédiate et révélatrice de certaines affinités sémantiques particulièrement éclairantes qui échappent souvent aux définitions elles-mêmes4 ${ }^{4}$. Si le mot doit être replacé en contexte(s),

4. Rappelons que l'importance des contextes et des discours figés a été particulièrement mise en évidence par J. Picoche. Voir entre autres : J. Picoche, 1992, Précis de lexicologie française, 
il convient également de prendre en compte les unités présentes dans les chaines dérivationnelles (famille de mots ou champs morphosémantiques) qu'il peut produire, comme par exemple hauteur, hautain ou bassesse. L'exploitation de toutes ces données (définitions, contextes, dérivés) conduit à établir une sorte de radiographie sémantique du mot dans laquelle on trouve à la fois les traits de base qui fondent sa signification et les représentations dominantes qui y sont associées.

Il n'est peut-être pas inutile de préciser ici la conception qui sous-tend cette utilisation du dictionnaire en vue de l'analyse de mots en discours. Il va de soi que nous ne considérons pas qu'il existe, pour un mot donné, un listage de significations préconstruites en langue et dont l'article de dictionnaire serait dépositaire. C'est au contraire le dictionnaire qui construit ses articles et ses définitions à partir des fragments de discours qu'il rassemble. Il en résulte que les articles lexicographiques, si on les lit bien, ne se réduisent pas à une suite de définitions données in abstracto, mais fournissent, avec les contextes qui révèlent les aptitudes combinatoires du mot, de véritables petits programmes de discours. Ces programmes présentent deux caractéristiques qui vont de pair : ils sont liés au contexte étroit du mot et relativement contraignants. Ainsi l'adjectif haut est vu à travers sa distribution immédiate, en tant qu'il s'attache à un support nominal auquel il confère une propriété. Il fournit ainsi autant de microdiscours qu'il rencontre d'unités avec lesquelles il se combine, comme par exemple hautes montagnes, voix haute, haut fonctionnaire, etc. Mais, même quand le contexte est largement ouvert, comme c'est le cas pour l'adjectif haut, il n'est pas pour autant illimité. Ainsi certaines combinaisons s'avèrent improbables (on parle de hauts faits mais moins de hautes actions) ou impossibles (*haute besogne, *haut commerçant)... L'intérêt du dictionnaire est précisément de nous faire découvrir ce noyau syntagmatique du mot, dans son étendue et ses limites, avec toutes les implications sémantiques qu'il contient, qu'il s'agisse des traits proprement définitionnels ou - et c'est peut-être là le plus intéressant - des traits subjectifs et évaluatifs. Toutes proportions gardées puisqu'on se trouve dans un cadre distributionnel minimal, on peut dire que l'énonciateur, quand il prend un mot dans le fonds lexical de la langue, ne s'empare pas d'une unité isolée grâce à laquelle il va dénommer telle ou telle chose, mais qu'il active, à travers ce mot, une pluralité de microdiscours

Paris, Nathan; 1992, "L'enseignement du vocabulaire en français langue maternelle au niveau de l'enseignement secondaire», Enjeux didactique du français, $n^{\circ} 26$, p.13-28 (paru aussi dans : 1995, Études de lexicologie et de dialectologie, CILF, p. 365-377). J'ai aussi exploité ce type d'approche dans l'étude du verbe crier (S. Rémi-Giraud, 2003, «Structuration interne de la polysémie du verbe crier», dans S. Rémi-Giraud et L. Panier (dir.), La polysémie ou l'empire des sens. Lexique, discours, représentations, Presses universitaires de Lyon, p. 111-128). Sur les collocations, on se reportera à F. Grossmann et A. Tutin (dir.), 2003, Les collocations. Analyse et traitement, Université Stendhal-Grenoble 3, «Travaux et recherches en linguistique appliquée ». 
à partir desquels il va pouvoir construire son discours propre. C'est ce qu'illustre de manière exemplaire l'emploi par J.-P. Raffarin de l'expression France d'en haut / France d'en bas.

\section{Haut et bas : la mise à distance}

Le Petit Robert fournit une seule entrée pour chaque unité mais avec trois identités grammaticales, adjectif, nom et adverbe - l'adjectif étant la catégorie première dont dérivent, par conversion, le nom et l'adverbe. Je traiterai d'abord de la polysémie comparée des deux adjectifs, en la présentant sous la forme très simplifiée du tableau page ci-contre 5 .

On voit clairement, par ce tableau, que la polysémie de ces deux antonymes suit un parcours parallèle. Le domaine spatial, qui implique la perception visuelle, est premier et regroupe les significations qui ont en commun d'exprimer les propriétés d'un corps $^{6}$ dans l'espace considérées par rapport à l'axe vertical. Ces propriétés sont de deux sortes : la dimension (l. 1.) et la position (I. 2.). La dimension «détermine la portion d'espace occupée par un corps» (Petit Robert) - sur l'axe de la verticalité dans le cas présent - et elle permet d'opposer ce qui est haut et bas, c'est-à-dire d'une dimension considérable (de hautes montagnes) ou peu considérable (table basse) par rapport aux objets de même espèce. La position concerne la «manière dont une chose, une personne est posée, placée, située» (Petit Robert). Cette position est également projetée sur l'axe vertical et évaluée par rapport à une autre position située sur cet axe. Trois cas se présentent selon que la position de l'objet est repérée par rapport à sa position normale (rester l'arme haute), par rapport à la position d'autres objets de la même espèce (plafond bas) ou encore selon qu'il s'agit de la position d'une partie de l'objet repérée par rapport à la position d'une autre partie de ce même objet (la ville haute, la basse ville, la plus haute marche du podium). La différence entre les deux significations (dimension/position) apparait clairement si l'on prend un exemple comme la plus haute marche, qui peut exprimer soit la dimension d'une marche qui serait au-dessus de la moyenne des autres (dans le cas d'un escalier aux marches inégales, par exemple), soit - et c'est le cas de l'exemple donné : la plus haute marche du podium - la position de la marche médiane de l'estrade, surélevée par rapport aux deux autres. Cette seconde signification est plus abstraite que la précédente si l'on considère qu'elle ne met pas en jeu directement la grandeur d'un

5. Je m’appuie sur le Nouveau Petit Robert (1993) et sur le Trésor de la langue française, tout en remaniant certaines des définitions proposées.

6. On notera que les adjectifs haut et bas ne peuvent se dire dans ce sens directement de l'humain ( ${ }^{*}$ un homme haut, bas) mais seulement de son physique (taille haute, front haut). 


\begin{tabular}{|c|c|}
\hline HAUT & BAS \\
\hline \multicolumn{2}{|c|}{ I. SENS SPATIAUX } \\
\hline \multicolumn{2}{|l|}{ 1. DIMENSION DANS LE SENS VERTICAL : } \\
\hline $\begin{array}{l}\text { - qui est d'une certaine dimension : } \\
\text { mur haut de deux mètres; } \\
\text { - qui est d'une dimension considé- } \\
\text { rable par rapport aux objets de } \\
\text { même espèce : } \\
\text { de hautes montagnes, homme } \\
\text { de haute taille. }\end{array}$ & $\begin{array}{l}\text { - qui est d'une dimension peu consi- } \\
\text { dérable par rapport aux objets de } \\
\text { même espèce : } \\
\text { table basse, voiture très basse. }\end{array}$ \\
\hline \multicolumn{2}{|l|}{ 2. POSITION DANS LE SENS VERTICAL: } \\
\hline $\begin{array}{l}\text { - qui est placé ou porté au-dessus } \\
\text { de la position normale ou } \\
\text { habituelle : } \\
\text { rester l'arme haute; } \\
\text { - qui se trouve situé au-dessus par } \\
\text { rapport aux choses de même } \\
\text { espèce : } \\
\text { la plus haute marche du podium; } \\
\text { - qui se trouve situé au-dessus par } \\
\text { rapport au reste de la chose: } \\
\text { la ville haute. }\end{array}$ & $\begin{array}{l}\text { - qui se trouve au-dessous } \\
\text { de la position habituelle : } \\
\text { les nuages sont bas; } \\
\text { - qui se trouve situé au-dessous par } \\
\text { rapport aux choses de même } \\
\text { espèce : } \\
\text { plafond bas, les branches basses } \\
\text { d'un arbre; } \\
\text { - qui se trouve situé au-dessous } \\
\text { par rapport au reste de la chose: } \\
\text { la basse ville. }\end{array}$ \\
\hline \multicolumn{2}{|c|}{ II. SENS FIGURÉS (DÉRIVÉS DE I. 2.) : } \\
\hline $\begin{array}{l}\text { - domaine temporel : } \\
\text { la plus haute Antiquité; } \\
\text { - domaine de la perception : } \\
\text { - sur l'échelle des degrés } \\
\text { d'intensité : } \\
\text { haute pression, haute fréquence, } \\
\text { haute tension; } \\
\text { - sur l'échelle des sons, des degrés } \\
\text { de puissance de la voix : } \\
\text { ton haut, notes hautes, } \\
\text { lire à voix haute; } \\
\text { - domaine financier: } \\
\text { le dollar est haut, hauts salaires. } \\
\text { - domaine de la puissance sociale } \\
\text { et politique : } \\
\text { hauts fonctionnaires, la haute } \\
\text { bourgeoisie, la haute société; } \\
\text { - domaine des valeurs intellectuelles, } \\
\text { esthétiques et morales: } \\
\text { haute intelligence, haute couture, } \\
\text { âme haute, hauts faits. }\end{array}$ & $\begin{array}{l}\text { - domaine temporel : } \\
\text { le Bas-Empire, la basse latinité; } \\
\text { - domaine de la perception : } \\
\text { - sur l'échelle des degrés } \\
\text { d'intensité : } \\
\text { zones de basse pression, } \\
\text { la température est basse; } \\
\text { - sur l'échelle des sons, des degrés } \\
\text { de puissance de la voix: } \\
\text { notes basses, à voix basse; } \\
\text { - domaine financier : } \\
\text { bas prix, bas salaires, les cours } \\
\text { (de l'or, de la Bourse) sont bas; } \\
\text { - domaine de la puissance sociale } \\
\text { et politique : } \\
\text { le bas peuple, le bas clergé, } \\
\text { basse origine; } \\
\text { - domaine des valeurs morales: } \\
\text { âme basse, sentiments bas, de } \\
\text { basses actions, basse complaisance, } \\
\text { envie, jalousie, basses besognes, } \\
\text { une basse vengeance. }\end{array}$ \\
\hline
\end{tabular}


corps, mais, pour un objet donné, le repérage de deux positions relatives l'une à l'autre sur l'axe de la verticalité.

Les deux adjectifs haut et bas possèdent cette double représentation de la dimension et de la position sur l'axe vertical, mais s'opposent par le fait que l'un dit le "plus» et l'autre le «moins» par rapport à une moyenne ou à une norme. Il est intéressant de constater que cette moyenne ou cette norme reste en quelque sorte implicite, n'étant pas lexicalisée (il n'y a pas de mot, pour dire la dimension ou la position moyenne, qui permette le repérage de ce plus et de ce moins) 7 . Enfin, ce qui différencie les deux adjectifs, c'est que haut possède la signification neutre "qui est d'une certaine dimension » (mur haut de deux mètres), qui n'a pas de corrélat du côté de l'antonyme bas : ce qui montre qu'il constitue le terme non marqué de l'opposition ${ }^{8}$.

C'est la signification relative à la position (I. 2.), donc la plus abstraite, qui est la source des nombreuses dérivations métaphoriques que l'on trouve en II, dans des domaines aussi divers que le temps, la perception (sonore, en particulier), la finance, la puissance sociale et politique, les valeurs intellectuelles, esthétiques et morales. Ce qui est transporté (au sens étymologique de métaphore), c'est la représentation de deux positions sur l'axe de la verticalité, en dehors de tout objet présent dans l'espace - les deux mots devenant alors disponibles pour dire la position d'entités non spatiales dont la plupart sont non physiques, abstraites. Là encore, il peut s'agir de la position d'une entité par rapport à sa position normale (haute pression, la température est basse, le dollar est haut), de la position d'une entité par rapport aux entités de même nature (hauts fonctionnaires, haute intelligence, hauts faits) ou de la position d'une partie de l'entité par rapport à l'autre (la plus haute Antiquité, la haute bourgeoisie, la haute société). Plus précisément, on peut dire que ce qui fonde la figure, c'est moins une analogie de propriété (comme le veut la présentation de métaphores classiques du type Pierre est un lion ${ }^{9}$, dans lesquelles il y a transfert d'un attribut typique du comparé au comparant) qu'une homologie de structure, comme c'est le cas ici ${ }^{10}$.

Dans le cas présent, cette structure présente des caractéristiques intéressantes. Elle permet de dire la verticalité (axe fondamental de nos représenta-

7. Il en est de même d'autres termes de dimension tels que long, large, épais (vs court, étroit, mince). Voir J. Goes, 1999, L'adjectif. Entre nom et verbe, Paris-Bruxelles, Duculot, p. 49; A. Borillo, 1998, L'espace et son expression en français, Gap, Ophrys, p. 59-62.

8. Là encore, il en est de même des adjectifs long, large, épais (vs court, étroit, mince).

9. Voir par exemple G. Molinié, 1992, Dictionnaire de rhétorique, Paris, Le Livre de Poche, «Les Usuels de Poche».

10. Sur les concepts de métaphore analogique et homologique, voir H. Constantin de Chanay et S. Rémi-Giraud, 2002, “"Espèces d'espaces” : approche linguistique et sémiotique de la métaphore », Mots, n 68, mars 2002, p. 75-105, et 2003, "Des ressorts, des bulldozers, des tremblements et des chapeaux : pour des tropes hors catégories", Morphosyntaxe du lexique. 2. Catégorisation et mise en discours. Travaux linguistiques du CERLICO, $\mathrm{n}^{\circ} 16$, Presses universitaires de Rennes, p. 179-203. 
tions, s'il en est), et, sur cet axe, une position saillante par rapport à la moyenne - l'ensemble se présentant comme statique. En tant que telle, elle offre une armature minimale et commode pour dire le positionnement de toutes sortes d'entités ${ }^{11}$. C'est sans doute ce qui explique l'extrême productivité en significations figurées de nos deux mots.

Mais il y a plus. Les antonymes haut et bas ne se contentent pas de désigner deux positions opposées sur cet axe vertical, ils attachent à ces positions des jugements de valeur que révèlent leurs combinatoires respectives. Comme on peut s'y attendre, le haut est valorisé tandis que le bas est dévalué ${ }^{12}$. On constate que le haut a un premier avantage au plan quantitatif : l'article haut est en effet nettement plus long que l'article bas, surtout en raison du corpus d'exemples, plus important pour haut. D'autre part, les significations figurées s'opposent d'un mot à l'autre. Du côté de l'adjectif haut se trouvent la force et l'intensité - la puissance de la voix pouvant conduire métonymiquement à l'expression de la véhémence (pousser les hauts cris) ou de la suffisance (avoir le verbe haut) - et surtout l'affirmation d'une supériorité dans la plupart des domaines de l'expérience humaine (argent, hiérarchie sociale et politique, valeurs intellectuelles, esthétiques et morales). L'adjectif bas récupère, lui, les valeurs négatives, en particulier dans le domaine social et le domaine moral où ce qui est bas est méprisable (le dérivé bassesse étant sans appel de ce point de vue) : les valeurs intellectuelles et esthétiques ne sont même pas représentées. Plus précisément, on observera certaines dissymétries instructives. Si les deux mots sont antonymes, leurs emplois ne forment pas pour autant des couples opposés. On constate une sorte de force d'attraction vers le haut qui fait que ce mot capte à son profit nombre de personnes et de choses - qu'il s'agisse de hauts fonctionnaires, d'une haute intelligence, de la haute couture, de la haute coiffure, de hauts faits (sans parler du Très-Haut!) dont on ne trouve jamais l'équivalent d'en bas... De ce point de vue, certaines affinités sont éloquentes : la haute bourgeoisie tient en regard le bas peuple, sans qu'aucune permutation adjectivale soit possible !

Mais la valorisation du haut ne va pas sans ambigüité. Si ce pôle est enviable, il ne suscite pas forcément une adhésion chaleureuse... On note d'abord que l'adjectif haut s'applique à l'administration (hauts fonctionnaires, la haute administration), à la magistrature (la haute magistrature), à la finance (la haute finance), aux institutions (les hautes sphères, la Haute Assemblée, la Haute Cour de justice), aux instances internationales (le Haut Commissariat aux réfugiés), bref à toute forme de pouvoir qui implique une hiérarchie statique

11. On notera dans les définitions du Petit Robert l'utilisation répétée du mot échelle (autre métaphore) pour dire cette structure abstraite.

12. Rappelons ici l'incontournable référence aux métaphores d'orientation de G. Lakoff et M. Johnson, 1985, Les métaphores dans la vie quotidienne, Paris, Minuit. 
et une dimension gestionnaire : c'est en vain qu'on chercherait *un haut commerçant, *un haut patron, *un haut entrepreneur, *un haut ministre, *un haut paysan, etc. ${ }^{13}$ D'autre part, le haut et le bas sont à distance l'un de l'autre. Vu d'en bas, ce qui est haut peut apparaitre comme peu accessible, hors de portée : cette fois, ce sont des expressions comme être à la hauteur, se montrer à la hauteur de quelque chose, placer la barre trop haut qui expriment la difficulté, l'effort par rapport à ce qui pourrait être trop haut. La conscience de la distance se mêle d'un sentiment d'incapacité, sinon d'impuissance. Mais quand on occupe la position haute, on a tendance à voir les autres de haut, à leur témoigner de la hauteur et de la condescendance, bref à être hautain - autant d'expressions et de dérivés qui disent cette fois la distance inverse, avec le sentiment de condescendance qui l'accompagne ${ }^{14}$. Ainsi la valorisation qui s'attache à l'adjectif haut est une valorisation «froide», qui tend à recouvrir éloignement et mépris.

\section{France d'en haut, France d'en bas : la proximité retrouvée}

L'expression France d'en haut / France d'en bas, en mettant en regard les deux mots haut et bas (emploi relativement rare, comme on l'a vu), joue parfaitement sur l'opposition - et la complémentarité - de leurs significations et de leurs valeurs. Cette expression dissocie en effet, dans ce tout qu'est la France, deux positions extrêmes (toujours sans position médiane) où se trouvent, d'une part, les dirigeants socialistes, présentés comme de "hauts» responsables distants et méprisants, et, d'autre part, les Français de «basse » condition, dévalorisés à la fois par leur statut social et par le mépris dont ils font l'objet. Ainsi se tient un premier discours dans lequel l'auteur disqualifie en fait ses ennemis et présente comme des victimes ceux dont il veut faire ses alliés.

Mais il ne suffit pas de creuser l'écart entre l'ex-pouvoir socialiste et les couches populaires qu'il n'a pas su convaincre, encore faut-il ramener cet électorat perdu à soutenir le gouvernement en place. Et pour cela, se rapprocher d'eux en tenant un discours de proximité.

13. C'est plutôt l'adjectif grand qui souligne la réussite de professions dynamiques ou créatrices (un grand patron, un grand artiste, un grand intellectuel, etc.) - l'adjectif gros marquant, lui, une sorte de réprobation devant l'expansion de professions jugées plus matérialistes (un gros commerçant, un gros paysan, etc.).

14. Les dictionnaires explicitent rarement ce type de représentations. On notera avec intérêt l'orientation nouvelle du Dictionnaire du français usuel, qui tend à intégrer dans les définitions certains points de vue que véhiculent les mots (J. Picoche et J.-C. Rolland, 2002, Dictionnaire du français usuel, Bruxelles, De Boeck-Duculot; voir l'article conjoint «haut et bas». 
C'est là qu'il convient de prendre plus précisément en compte la forme de l'expression France d'en haut / France d'en bas, dans laquelle on trouve les deux locutions adverbiales en haut et en bas. Ces locutions, construites sur les adjectifs substantivés (le) haut et (le) bas ${ }^{15}$, entrainent un changement de catégorie grammaticale qui n'est pas sans importance, dans la mesure où il introduit les acteurs concernés dans une perspective topologique. L'en bas s'oppose alors à l'en haut comme la terre s'oppose au ciel : les expressions ce bas monde, ici-bas ne désignent-elles pas, elles aussi, notre monde terrestre? Du coup, par un retournement axiologique inespéré, ceux d'en bas héritent de toutes les représentations qui s'attachent à notre présence dans ce monde d'ici-bas : le sens des réalités (voir l'expression avoir les pieds sur terre), de l'expérience concrète, des difficultés de la vie... Par effet de retour, ceux d'en haut ne peuvent être que dans les nuages, dans un monde abstrait, détaché des réalités (où, en principe, on plane) - autant de traits qui viennent en renfort de la distance méprisante dont ils étaient déjà crédités.

Ainsi la situation se trouve-t-elle inversée. Si les mots haut et bas sont porteurs de valeurs opposées qui donnent à première vue l'avantage à la position haute par rapport à un bas dévalué, on peut dire que l'expression France d'en haut / France d'en bas retourne comme un gant cette axiologie première. En jouant simultanément sur la valorisation «froide» qui s'attache à l'adjectif haut et sur la perte du sens des réalités qu'implique la locution en haut, cette expression dénonce l'arrogance et l'irresponsabilité des dirigeants socialistes qui se trouvent ainsi doublement disqualifiés. Parallèlement, la position basse, qui n'est dévaluée que par le regard de ceux qui l'ignorent (à tous les sens du terme) en la considérant d'en haut et de haut, se trouve réhabilitée par les qualités implicitement attribuées à ce monde d'en bas, où se trouvent ceux qui affrontent au quotidien les dures réalités de la vie ${ }^{16}$.

Reste le plus difficile pour un Premier ministre : tenir à cette France d'en bas un discours de proximité qui le démarque de ses adversaires politiques.

Certes, Jean-Pierre Raffarin est indiscutablement un homme d'en haut du point de vue social et politique. Fils d'un ancien secrétaire d'État à l'agriculture, ancien dirigeant marketing d'un grand groupe privé qui a commencé sa carrière dans les cabinets giscardiens, sénateur de la Vienne, ministre des PME dans le gouvernement d'Alain Juppé, il ne peut jouer sur des origines populaires ou

15. Voir Littré : «En haut, loc. adv. Dans le lieu qui est le plus haut. En bas, loc. adv. Dans un lieu placé au-dessous.»

16. J'ai relevé avec intérêt qu'un élu communiste (Paul Fromonteil) reprend l'expression de J.-P. Raffarin en rétablissant (spontanément ?) les adjectifs (substantivés) le haut/le bas : "Je crois qu'on ne peut pas gérer les Français du “bas” si on n'intervient pas sur ceux du "haut” » (cité dans Le Monde, 11 mai 2002, p. 9) - ce qui redonne à bas sa charge de mépris en la renvoyant à son auteur. 
une ascension sociale laborieuse. Mais si l'on consent charitablement à mettre au second plan ce type de considérations, on peut mettre l'accent sur certaines de ses qualités personnelles, qui, telles du moins qu'elles apparaissent à travers les paroles et les comportements affichés, militent en faveur d'un rapprochement possible avec le monde d'en bas. Pour cela, il faut impérativement que ces qualités soient présentées comme celles d'un «terrien ». Servi par une silhouette plutôt massive (" "J'ai mes rondeurs", dit-il» ${ }^{17}$ ) et peu soucieux du dernier détail vestimentaire à la mode (" “Il n’a pas de revers au pantalon”, s'exclame Le Temps de Genève ${ }^{18}$ ), le Premier ministre se présente avant tout comme un homme de terroir et un homme de terrain. Homme de terroir, il en donne incontestablement l'image à travers l'affirmation répétée de son appartenance au Poitou-Charentes, sa région d'origine, et de son attachement à la province et à la campagne. Mais plus encore, il se veut homme de terrain, lui qui, en 1996, alors qu'il était ministre des PME, du commerce et de l'artisanat, donnait déjà des preuves de cet engagement au plus près de ses administrés, comme le laissent entendre ces lignes acidulées de Pierre Georges :

Au motif, irréfutable, qu' « un ministre de terrain doit aller sur le terrain » [je souligne], cet homme d'humus et de convivialité arpenteuse décida donc qu'une semaine par mois son ministère et lui-même se décentraliseraient en province. Qu'une semaine par mois, donc douze semaines par an, Jean-Pierre Raffarin et son équipe de la rue de Lille, siège du ministre itinérant et circumpolaire, s'en iraient prêcher, débattre et décider avec les terriens du terrain. (Le Monde, 8 mai 2002, p. 47) ${ }^{19}$

Lui qui, à peine élu, revendique la primauté du terrain sur l'affirmation abstraite des grands principes, évoquant en ces termes la République :

Pas une République qui se contente de Liberté-Égalité-Fraternité tout en haut sur nos mairies, mais sur le terrain. (J.-P. Raffarin, cité dans Le Monde, 9 mai 2002, p. 2)

Lui qui, enfin, se rend quasiment du même pas dans une exploitation agricole à proximité de Rennes, se présentant en messager de la ruralité qui mesure le blues rural en France ${ }^{20}$. Tout l'oppose donc à ces socialistes froids et distants, parfaits représentants d'une haute administration déshumanisée, comme le note Der Spiegel :

Le nouveau chef du gouvernement est le contraire du technocrate élitiste cultivé sous serre, qui prospère dans l'administration centralisée à la française. (Le Monde, 22 mai 2002, p.14)

17. Cité dans Le Monde, 9 mai 2002.

18. Cité dans Le Monde, 22 mai 2002.

19. Par-delà la figure du Premier ministre, G. Courtois parle de «génération terrain » pour les nouveaux élus des législatives de juin 2002 (Le Monde, $23-24$ juin 2002, p. 15) - expression déjà employée pour les municipales de 2001.

20. Cité dans Le Monde, 31 mai 2002, p. 11. 
Tout ? Il est encore une vertu à ne pas négliger. Ả l'arrogance et au mépris, à la condescendance qui rabaisse l'autre, il faut répondre par la plus parfaite modestie - cette dernière qualité venant parfaire l'image d'un homme proche de la France d'en bas, comme le résument ces lignes narquoises de Dominique Dhombres :

Un homme rond, venu de sa province, plein de bon sens, et pas fier avec ça. Un Français moyen. (Le Monde, 17 décembre 2002) ${ }^{21}$

Certes, Jean-Pierre Raffarin n'appartient pas à un milieu social modeste, mais il incarne cette qualité au plan moral - la polysémie du mot se prêtant parfaitement à ce glissement d'un domaine à l'autre... ${ }^{22}$

L'approche lexicale permet de mieux comprendre le double discours d'éloignement et de proximité qui se loge dans l'expression France d'en haut / France d'en bas. Le discours (ou, du moins, le point de vue) d'éloignement, imputé à l'adversaire et implicitement dénoncé, joue sur l'opposition entre un haut valorisé et un bas dévalué, censée représenter la distance qui sépare les dirigeants socialistes des couches populaires qu'ils méprisent. Le discours de proximité, qui est l'apanage de l'énonciateur, se fonde sur la réhabilitation du monde d'en bas et des valeurs de réalisme et de solidité qui s'y attachent; il trouve son accomplissement dans l'image en miroir d'un ministre terrien - provincial, modeste et au plus près des réalités... En même temps, ces deux discours révèlent et exploitent la propension qu'ont les mots à glisser d'un domaine à un autre. Si l'expression France d'en haut / France d'en bas exprime en principe un clivage social, elle capte à son profit toutes les représentations qui, au plan moral ou psychologique, s'attachent aux mots haut, bas, à leurs dérivés et aux expressions qu'ils forment. Ainsi, entre l'arrogance des élites et le réalisme de ceux qui luttent au quotidien, entre la distance méprisante des anciens dirigeants et la modestie revendiquée du nouveau chef du gouvernement, ce sont les qualités personnelles, les comportements, les attitudes psychologiques qui se trouvent mis en avant et nous introduisent dans un monde subjectif et moral qui masque la dimension proprement politique du débat.

Mais, quelles que soient les opinions, le lexicologue ne peut que rendre grâce à l'auteur de l'expression France d'en haut / France d'en bas pour l'éclairage qu'elle apporte sur le fonctionnement des mots en discours. Si l'on récapitule l'étude qui vient d'être faite, on voit que ce fonctionnement peut être

21. Voir le portrait que fait J.-B. de Montvalon de J.-P. Raffarin dans Le Monde des 23-24 juin 2002 ( UUn atout nommé Raffarin », p. 14). Voir aussi l'article de Ph. Ridet, «Un dessein modeste pour la France» (Le Monde, 16-17 juin 2002, p.1 et 21), qui ironise sur le modeste/modestie et qui rappelle que dès le lendemain de sa réélection, "Jacques Chirac avait souhaité un gouvernement "modeste, cohérent et efficace" » [je souligne].

22. On notera la tendance du lexique à euphémiser l'expression de la "pauvreté » par des mots empruntés au domaine moral (les gens modestes, simples). 
décrit à trois niveaux ${ }^{23}$. À un premier niveau, on peut dégager, en observant la totalité du parcours polysémique du mot, les traits sémantiques qui structurent l'ensemble des significations et fondent son unité sémantique. Pour le couple d'antonymes haut/bas, la polysémie conduit des sens spatiaux (dimension et position) à la multiplicité des significations métaphoriques (dérivées du sens positionnel). De ce double parcours, on peut extraire les traits suivants (communs aux deux adjectifs) : verticalité, dimension/position selon que l'axe de la verticalité sert de mesure ou d'échelle (à des positionnements distincts), dépassement d'un seuil (moyenne ou norme). Haut et bas s'opposent par le sens de ce dépassement, qui va au-dessus ou au-dessous du seuil, disant ainsi, pour l'un, la quantité/valeur positive (haut), et pour l'autre, la quantité/valeur négative (bas). À ce niveau, qui est le plus abstrait ${ }^{24}$, on peut dire que les deux adjectifs sont en quelque sorte en miroir l'un de l'autre. Le deuxième niveau est celui des contextes d'emploi du mot, qui conditionnent l'apparition des différentes significations. Mais au-delà de cette fonction de sélection (ou de stabilisation) du sens, ces contextes, entendus au sens large (syntagmes, collocations, expressions figées), nous livrent autant de microdiscours riches en représentations et valeurs associées. C'est l'observation de ce niveau ${ }^{25}$ qui révèle la valorisation froide de l'adjectif haut, sa charge de mépris, ainsi que les valeurs axiologiques opposées du bas et de l'en bas. C'est là que s'installent les dissymétries de fonctionnement, non prévisibles à partir du couple haut/bas pris abstraitement - mais fort instructives. Ainsi l'adjectif haut permet-il de construire nombre de syntagmes et d'expressions auxquels ne répond aucune formation antonymique avec bas... Le troisième niveau, enfin, est celui du discours particulier auquel on a affaire. À ce niveau, l'énonciateur introduit le mot dans le contexte d'emploi qui correspond à sa propre visée de discours. En même temps qu'il sélectionne (ou stabilise) la signification du mot, il peut activer (et/ou renouveler) les représentations présentes dans les microdiscours qu'il reçoit de ce mot. L'expression France d'en haut / France d'en bas est exemplaire de ce point de vue. On remarque d'abord que cette expression rétablit entre les deux antonymes une symétrie dont on a vu qu'elle était relativement peu fréquente dans l'usage. Cette opposition mise

23. On notera que B. Victorri propose un modèle de la polysémie qui comporte également trois niveaux. Toutefois, le deuxième niveau de son modèle n'est pas assimilable à celui que nous préconisons (voir B. Victorri, 1997, "La polysémie : un artefact de la linguistique ?», Revue de sémantique et de pragmatique, $\mathrm{n}^{\circ} 2, \mathrm{p} .41-62$ ).

24. C'est à ce niveau qu'on peut parler d'un invariant, constitué de traits sémantiques mais sans corrélat référentiel, subsumant les différentes significations du mot. Une synthèse a été faite par G. Kleiber des diverses approches (citons entre autres C. Fuchs, B. Victorri, P. Cadiot, F. Nemo, J.-J. Franckel, D. Paillard...) qui tentent actuellement de théoriser la problématique de la polysémie à partir de conceptions aréférentielles du sens (G. Kleiber, 1999, Problèmes de sémantique. La polysémie en questions, Lille, Presses universitaires du Septentrion).

25. Que vient éventuellement enrichir la prise en compte des dérivés au plan morphématique. 
en place, l'auteur peut alors jouer à la fois sur l'axiologie positive et négative de haut, sur le renversement des valeurs du bas et de l'en bas, pour tenir le double discours de la distance méprisante (attribuée aux socialistes) et de la proximité rassurante (des terriens auxquels il s'assimile). Si la réflexion théorique tend à privilégier le premier niveau d'analyse, il est clair que c'est le second niveau - celui de la combinatoire - qui s'avère le plus pertinent pour l'étude des mots en discours, l'énonciateur devant toujours négocier l'emploi qu'il fait d'un mot avec les microdiscours dont ce mot se trouve implicitement chargé.

L'expression France d'en haut / France d'en bas a certes été entendue ${ }^{26} . .$. et étendue, bien au-delà du message qu'elle voulait communiquer. Tout s'est passé, semble-t-il, comme si, à travers ce jeu des contraires, tout un chacun s'avisait de cette évidence qu'il y a en toutes choses un haut et un bas en relation d'opposition. Ainsi a-t-on vu fleurir une gauche d'en haut / une gauche d'en bas ${ }^{27}$, un PS d'en haut ${ }^{28}$, une UMP d'en bas ${ }^{29}$, des élus d'en haut / des élus d'en bas ${ }^{30}$, des ministres d'en bas ${ }^{31}$, des mesures d'en bas ${ }^{32}$, une CGT d'en haut / une CGT d'en bas ${ }^{33}$, des entreprises d'en haut / des entreprises d'en bas 34 , jusqu'à un terrorisme d'en haut / un terrorisme d'en bas 35 - sans compter la descente aux étages inférieurs (de la France du sous-sol, par exemple ${ }^{36}$ ) - échappés, comme autant de bons mots, de la boite de Pandore ouverte par notre Premier ministre...

26. Échappant à une néologie transitoire, elle se trouve déjà consignée dans l'édition de 2003 du Nouveau Petit Robert.

27. Olivier Besancenot (cité dans Le Monde, 31 mai 2002, p.12). Voir aussi l'article d’É. Fottorino dans Le Monde, 8 octobre 2003, p. 36.

28. Gérard Collomb (cité dans Le Monde, 16 octobre 2002, p. 9).

29. Entendu au Journal de 20 heures de France 2, d'un député de l'UMP dans l'Aisne qui distribuait des tracts sur l'École.

30. Un ministre, sous le sceau de l'anonymat, lors des Assises des conseillers généraux à Strasbourg, le 31 octobre 2002 (Le Monde, 3-4 novembre 2002, p. 6).

31. P. Georges, Le Monde, 23 novembre 2002, p. 36.

32. Ch. Albenque, syndicaliste CFDT, cité dans Le Monde, 10-11 novembre 2002.

33. J.-P. Raffarin vantant «les mérites de la régionalisation du transport ferroviaire» (cité dans Le Monde, 26 octobre 2002).

34. Le Monde, 12 février 2003.

35. Entendu sur Europe 1, 18 mars 2004, entre $9 \mathrm{~h} 30$ et $10 \mathrm{~h} 30$.

36. Ph. Val, France Inter, 3 juin 2002, 18 h 55. 